\title{
THE PARADIGM OF COMPUTER INTEGRATED CONSTRUCTION APPLIED TO CIVIL-ENGINEERING
}

\author{
François Peyret
}

\author{
Site Robotics Subdivision, Laboratoire Central des Ponts et Chaussées, \\ B.P. 19, 44340, Bouguenais, France
}

\begin{abstract}
The main issue which has to be addressed, when one considers the robotisation of civil engineering tasks, is the paradigm of computer integrated construction. This paper first stresses the advantages that a common digital data base, managing all the geometry of the structure all along its construction, could bring. Then, it analyses the state-of-the-art solutions that can be used for equipment positioning, which is the common technological key to all the applications, with reference to the different families of machines. Finally, are described some products or projects which proceed from that paradigm of computer integrated construction.
\end{abstract}

Keywords : robotics, civil engineering, computer integrated construction, positioning, GPS

\section{INTRODUCTION : CIVIL- ENGINEERING AND ROBOTICS}

Compared to building construction industry, civil engineering construction is highly mechanised. Very few tasks are performed manually, several cubic meters of soil cannot be removed by hand as well as a 30 meter deep hole in the rock cannot be drilled by hand. That is why huge machines as bull-dozers, scrapers, shovels, excavator, etc., are the main workers of a civil engineering sites. It is true that cranes and concrete pumps are also present on building sites, but all around them are acting an army of human workers, achieving numerous and various manual tasks which robotisation appears clearly now to be achievable only in fantasy books.

For big civil engineering sites, for instance openpit mining, airport tracks or motorway construction, dam construction, etc., the manual works can be reduced to almost nothing. In those conditions, from the actual pieces of equipment to civil engineering robots, remain mainly electronics and software issues to handle, the mechanical structure being already existing.

But the real question is now : which kind of robots ? Referring to the major publications in the field of robotics, nobody will be shocked if we assume that there are to main different categories of machines which we usually call robot : the autonomous or remotely controlled ones and the smart ones. The "robot kings" are both.... How should be our robotised bulldozers ? Definitely smart and non autonomous, except the very rare cases where they have to dismantle a nuclear plant or when they are working right at the bottom of a volcano ready to burst, i.e. when it is really unlike to send human beings... It seems quite obvious now, to most of the people, that spending enormous amounts of money trying to remove the human drivers from the machine has no real sense, neither from an economic point of view, nor from a social one.

A smart machine is a machine which has a little bit of sensing capabilities, a little bit of memory and a little bit of intelligence to fulfil the mission it has been programmed for in its changing environment. This mission being generally formulated in terms of geometry, it is clear that the key sensing technology which is concerned that the positioning technology. Even more than the key technology for the robotisation of the construction tasks, accurate and real-time positioning can also bring to construction industry the possibility to link the different phases of the global process, i.e. to enter into the time of "Computer Integrated Construction", which is definitely the true issue.

\section{WHY COMPUTER INTEGRATED CONSTRUCTION?}

A civil engineering structure as a bridge, a dam, an embankment or a road, is like any manufactured object, it has to be designed, that is to say its characteristics are to be established, in order to provide the services it has been intended to provide.

Among those characteristics are in particular its shape, composition and relative dimensions, as any other object, but also, what manufactured objects do not need, its exact location with reference to the environment in which it should take place. 
For instance, everybody has seen on the roadworks he was driving along, the forests of multicoloured wooden stakes, indicating where the future road would be built in the coming months. These pegs will be used all along the works as images of the project, as the almost only residual data coming out of the design office.

Surveyors will set up, manage and control these reference points. First, they will materialise the "vertebral column" of the road, often called "red line", generally the central axis of it. Then, they will materialise the width of it, especially the alignments where the ground should be cut off or brought during the earthworks phase, or the alignments inside of which the materials should be laid during the pavement construction phase. Additional elevation indications on the pigs, as nails or painted marks (using various codes of colours) are also present on some of them, providing information on where the final level should be. Finally, the most sophisticated ones are those called "chairs", which are composed of 3 staffs, assembled in triangle, where the hypotenuse is supposed to represent the slope of the embankment they are delimiting the contours of.

These stakes will be used all along the works by the various pieces of equipment which will cut, fill, spread and compact the different layers of the road, as physical references to guide them. For that purpose, different methods, more or less humancontrolled, but not really "high-tech", are traditionally used. One can use some kind of rope stressed between two stakes, to get the right level of the road at this section, or some kind of optical line coming out of the eye of the operator and leaning upon two targets placed at the right level by two other colleagues...

These traditional tools and methods are indeed skilfully used since our roads do not break into pieces after one year of traffic, but what is also clear, is that, at the starting of the third millennium, there should be more effective and reliable means of handling the geometrical data.

The paradigm of computer integrated construction aims at providing a new methodology, based upon the sharing of a common numerical geometrical data base from the design, through all the site operations up to the quality control of the geometry of the structure. It relies upon the main following technologies : Computer Aided Design (CAD) software tools, automatic control and particularly, the key technology that was the latest to be developed, the real-time positioning.

Real-time positioning creates the link between all the phases of the design-construction-assessment process in the sense that it provides to any agent (human or non-human) the capability to refer his actual work, i.e. the position or trajectory of his tool, to the target work which should be achieved. By this comparison between target and actual position, in the same reference system, the automation becomes possible, at any level, from a simple operator-aiding system to the fully autonomous system [1] [2].

\section{THE BASIC ARCHITECTURE OF A COMPUTER INTEGRATED CONSTRUCTION (CIC) SYSTEM}

A CIC system, excluding the CAD system which can be considered at the moment as a design-only tool, is typically composed of three main sub-systems

- a ground sub-system, which is the interface between the CIC system and the amont and aval tasks of the complete construction process,

- a positioning sub-system, which will provide the system with the necessary position data,

- an on-board sub-system which controls all the functions supposed to be realised by the system on the machine itself.

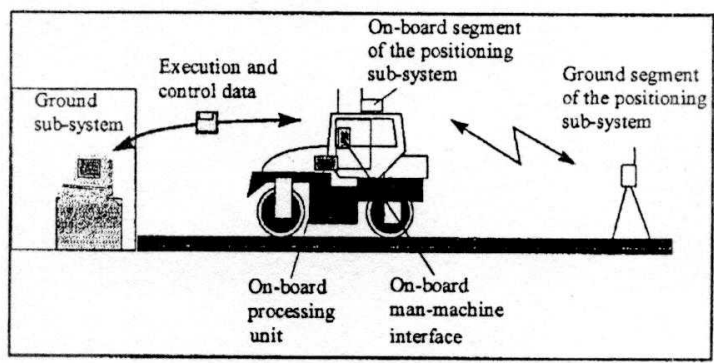

Fig. 1: The basic architecture of a CIC system

\subsection{The ground sub-system}

It is a pure software tool, hosted by a standard computer which is generally located on the site. Its rôle is absolutely fundamental, despite the fact that it is not as "visible" as the on-board sub-system on the machine.

Its main functions are :

before the execution of the work :

- to translate the geometrical data coming from the $\mathrm{CAD}$ into data formatted for the CIC application and to create the geometrical data base which will support all the CIC functions,

- to allow the user to add manually some data which are established at the level of the work site itself, not necessary included in the $\mathrm{CAD}$, and needed by the CIC system for its operation,

- to allow the user to define the "mission", that is to say to select from the global numerical data base the part that will be considered by the on-board application as the target work for the day,

- to provide the user with a mean to transmit this mission to the on-board sub-system,

after the execution of the work :

- to provide the user with a mean to collect the achieved work (currently called "as-built") data coming out from the on-board sub-system, 
- to update the geometrical data base,

- to allow the user to post-process these data, the way he wishes to do it,

- to provide the user with a mean to export these data when they are needed by another application.

\subsection{The positioning sub-system}

This sub-system is indeed the key-module of all the CIC systems, the one for which the technological developments were the most expected.

It is generally a real system, in the sense that it is not a single instrument. It is composed of various sensors and emitters, depending of the specifications, the constraints and the chosen basic technology.

Its main function is unique and consists in providing the on-board sub-system with the position and attitude information required by the application. There are numerous different specifications. For instance, a drilling machine will in the absolute need 6 independent position parameters fixing the position in space of its beam. These can be the co-ordinates of one point of the tool and three angles defining its orientation as well as two sets of co-ordinates of two different points. In some cases, when some of them are already defined by other means, this number can be reduced to five, even less, depending on the degree of automation expected. The case of a paving machine is very similar, its tool being also analogue to a rigid beam, this time rather horizontal, but needing also to be totally localised (i.e. in terms of position and attitude) in space. These kind of applications are indeed the most demanding in terms of number of parameters and in terms of accuracy. On the other hand, some machines, as compactors, require only two parameters, which are its plane $2 \mathrm{D}$ position, needed to control the distribution of its compacting energy.

\subsection{The on-board sub-system}

This sub-system is generally the most complex one, composed of both hardware and software, and can be divided into several physical parts.

Its main functions are generally:

- to allow the user to enter the mission data coming from the ground sub-system,

- to process the inputs from the positioning subsystem,

- to inform the driver upon its location on the site,

- to inform the driver upon the work supposed to be achieved at this place (generally a target surface to obtain), upon the actual position of its tool and upon the gap existing between them, together with some visual indication to help him to reduce this gap,

- to record the work achieved (as-built data),

- to allow the user to transfer the data to the ground sub-system.

\section{WHAT ARE THE SUITABLE POSITIONING TECHNOLOGIES}

\subsection{Introduction: the concerned machines}

Numerous positioning technologies exist and can be combined to give a quite infinite number of solutions. Actually, the choice of a particular solution will depends upon many different criteria among which the technical one may not be the main one. Most of the time, the final choice is a compromise between capabilities of fulfilling the requirements specifications, connected operational constraints and cost, made inside the set of feasible techniques. In this chapter, we will argue only about technical and operational issues, with the background idea however that we are not envisaging totally out-of-rangesolutions from a financial point of view.

These two issues, technical and operational, will be discussed for civil-engineering machinery. Before that, to be as clear as possible, we need to describe a little more precisely what kind of machines we are talking about, to say for what kind of construction task they are currently used and to propose a simple general classification, from a localisation requirements point of view. We propose the above classification, using the following convention for the needed accuracy : $\mathrm{H}$ (high) for centimetre accuracy, $M$ (medium) for decimetre accuracy and L (low) for metre accuracy.

\section{Earthmoving and mining machines}

They currently have to displace large amount of soil or ore, needing that way to locate their tool in 3D $(\mathrm{X}+\mathrm{Y}+\mathrm{Z})$, accuracy $\mathrm{M}$, in order to manage volumes. They may move at relatively high speed even when they are working, i.e. a few kilometres per hour, let us say up to $10 \mathrm{kph}$. Among them are bull-dozers, shovels, wheel-loaders, excavators, scrapers, graders (in their spreading works). Their working speed is between 5 and $10 \mathrm{kph}$, except shovels and excavators which work stationary. Dump trucks do not belong to this family and will not in fact be taken into account since they perform only transportation tasks.

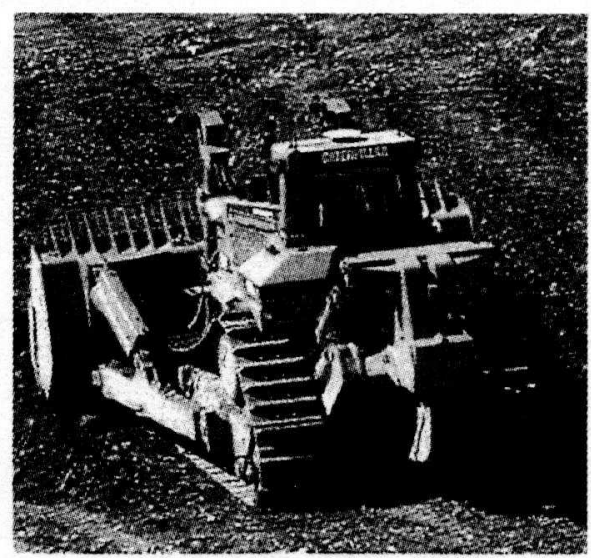

Fig. 2 : One earth-moving machine: the bulldozer 
Drilling machines

They are characterised by the fact they are stationary when they are working and that their tool is roughly vertical. They require $6 \mathrm{D}$ positioning, accuracy H-M, when they are working and $3 \mathrm{D}$ $(\mathrm{X}+\mathrm{Y}+\mathrm{yaw})$ positioning, accuracy $\mathrm{M}$, when they are travelling from one working point to another.

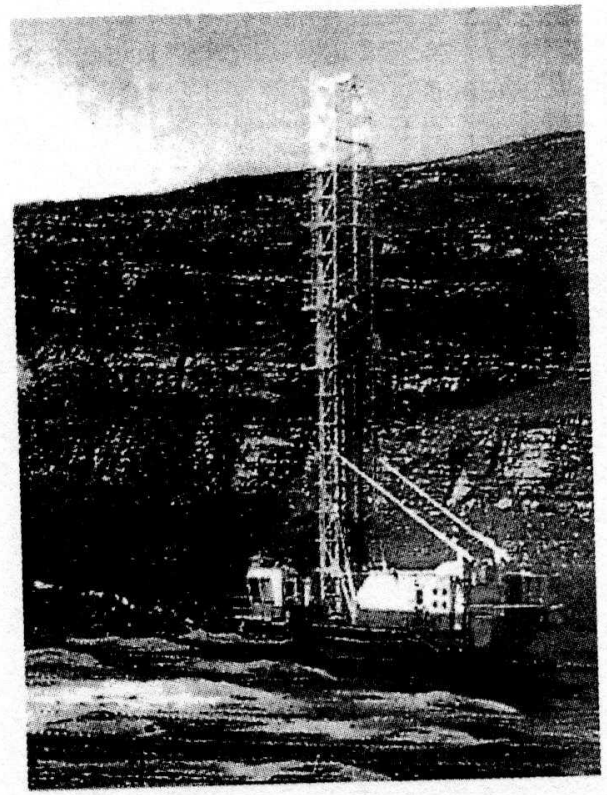

Fig. 3 : One drilling machine

\section{Profiling machines}

So-called machines are those which are finishing the surface of the course under process by imposing them a given profile, both longitudinal and transversal. For that, they need to know and to control the position and attitude of their tool, and consequently to be equipped with a $5 \mathrm{D}$ or $6 \mathrm{D}$ positioning device, accuracy $\mathrm{H}$. Their working speed is relatively low, of a few mps. In this group can be considered pavers (for concrete or asphalt), milling machines, autograde or graders when they are performing fine final grading.

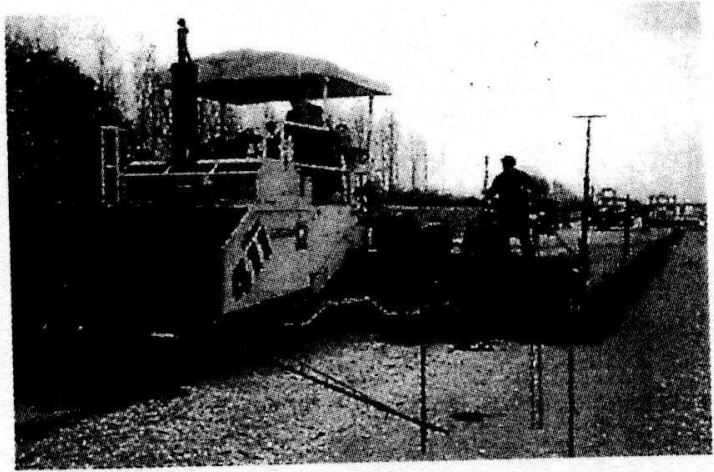

Fig. 4 : One profiling machine: the asphalt paver

\section{Surfacing machines}

We call surfacing machines the pieces of equipment which don't care of the elevation, that is to say work on a surface, to process it, either to compact or to $\mathrm{mix}$ it with cement or... Their positioning requirements are only $2 \mathrm{D}$, accuracy $\mathrm{M}$. Their working speed is between 5 and $10 \mathrm{kph}$, as the earthmoving group. In this group are essentially the compactors, whatever they compact, and the soil treating machines.

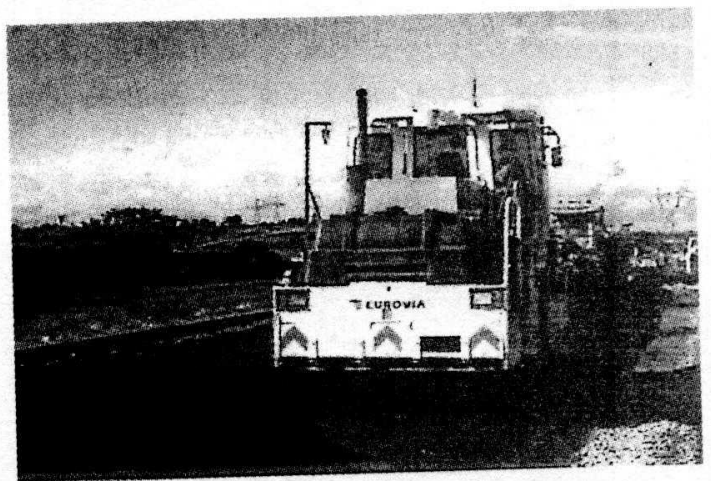

Fig. 5 : One surfacing machine: the compactor

\subsection{Technical issues}

Functional and global design of the positioning subsystem : specification of the configuration

As we said previously, the first step when considering the automation of a civil-engineering piece of equipment is the good specification of the need in terms of positioning. This one is strongly dependent upon the level of automation which is targeted. For instance, a paving machine would need only four parameters if you aim only an automatic control of the screed : the plane co-ordinates $\mathrm{X}$ and $\mathrm{Y}$ (to recover the target surface it is suppose to pave at its current position) and the actual position of the screed in the vertical plane perpendicular to the horizontal projection of the alignment of the road. This latest position is determined by two altitudes $Z_{1}$ and $\mathbf{Z}_{2}$ or one altitude $\mathbf{Z}$ and one cross-slope angle $\mathbf{c}$.

In some cases, the two parameters $\mathbf{X}$ and $\mathbf{Y}$ (representing the plane co-ordinates of a reference point of the screed, for instance the origin of the mobile frame) are already linked by a relation which is the theoretical equation of the horizontal alignment of the road, making the assumption that the machine does actually follow this alignment. In this case, a single parameter, generally the curvilinear abscissa $s$ can replace the two previous ones. From a practical point of view, this apparent simplification is not a real one since reliable sensors for measuring a curvilinear abscissa are not so easy to find.

To continue our example about the paver, if this time the targeted level of automation is the elevation control of the screed and the steering of the tractor itself, another position parameter need to be added to the four previous ones is the horizontal direction angle, also called the 'yaw' angle $\mathbf{d}$.

The global design of the positioning sub-system is also strongly dependant of the physical constraints 
of the application. In some environments, some technical choices are forbidden (ex.: GPS in tunnelling) and some others have to be envisaged where they would not have been necessary in other environments. One example is the need to use deadreckoning sensors in addition to the GPS receiver, in GPS has been chosen as the main solution, in road construction environments when bridges create artificial masks between equipment and satellites.

Some emerging technologies with reference to the different types of equipment

Earthmoving, mining machines and drilling
machines

The GPS technology, used in its suitable mode (real-time kinematic or "RTK" mode) seems to be very convenient given the level of accuracy required. In the cases when the environment is too obstructed for the satellites, ground-based systems using millimetre waves must be envisaged, with an adequate placement of the beacons. In no case optical systems are convenient given the relatively high speeds and travelled distances. For drilling machines, during the drilling process itself, it is necessary to add appropriate inclinometers for the control of the attitude angles of the boom.

\section{Profiling machines}

These machines are very demanding in terms of accuracy. For that reason, current GPS solutions are not convenient for the moment. Optical technologies are suitable, although the range is generally limited to a few hundred of metre, given the low progress speed which leave the time to the surveying teams for displacing the equipment [3]. The instrument called "robotized total station" or RTS, capable of tracking a prism mounted on the machine and providing its $3 \mathrm{D}$ co-ordinates, is one example of a good positioning solution for that family of equipment, needing however also inclinometers for the determination of the attitude angles.

More sophisticated novel systems, still opticalbased but capable of providing the 6 degrees of freedom of the mobile they are attached to, can also be envisaged, such as Odyssey TM from SPSi or Laserguide from the University of East London [4], still under development.

\section{Surfacing machines}

As for the earthmoving machines, GPS seems to be the suitable technology. Nevertheless, the tricky point is that the machines of this family which are used for road building, often have to work under bridges and suffer from masking phases of the GPS satellites. The "on-the-fly" re-initialisation time of the RTK GPS systems still being of the order of one minute, the travelled distances before recovering the decimetre precision required may be important at the proximity of bridges. To prevent from that, additional sensors have to be added to perform deadreckoning navigation during the "blind" periods of the GPS system. The most convenient deadreckoning sensors seem to be optical fibre gyrometers and Doppler radars as odometers [5].

Some other optical solutions may be envisaged, such as those performing $2 \mathrm{D}$ triangulation using a rotating laser beam mounted on the vehicle and reflective bar-coded targets in the environment.

\subsection{Operational issues}

From a practical point of view, each positioning technologies suffers from structural drawbacks that may prove to be too constraining in the daily use.

For instance, the systems needing numerous targets all along the road site may be unacceptable by the contractor, given the too important manpower required. It can be the same for the solutions using instruments on the ground that have to be displaced and re-initialised regularly with a tricky and timeconsuming procedure.

The sensitivity to physical and climatic conditions is also an important parameter to be considered. From this point of view, optical solutions may be disadvantaged compared to Hertzian waves ones as GPS.

\subsection{The challenge: GPS for everything}

GPS technology benefits from two major advantages, which are first to be global and secondly to be $3 \mathrm{D}$.

The first advantage gives to him what we can call the "temporal coherency", meaning that the reference system remaining the same, measurements performed with a GPS receiver will remain coherent whatever the time they are executed.

The first advantage can be called the "spatial coherency" and means that the three dimensions are intrinsically linked inside the GPS positioning process, compared to other solutions where they may be obtained through independent ways.

These two important features are absolutely requested from the positioning system which is utilised inside a CIC application, since they are needed to guarantee a perfect coherency between the co-ordinates from the design, to be achieved, and the co-ordinates measured on the site by the system.

GPS is indeed the only system gathering so well these two advantages. However, it suffers from some specific drawbacks which still limit its development on the civil engineering sites : the lack of accuracy when coming to centimetre, the weak availability that may be critical in some zones and the constraints linked to the base station necessary to the differential mode.

When these three main drawbacks are overcome, RTK GPS will surely become a solution suitable to almost any kind of civil engineering task. Some encouraging tracks for solutions already exist [6]. 
The accuracy is improving year after year, thanks to the improvement of both hardware and software components, especially in the domain of multi-paths rejection, electronic noise reduction and on-the-fly ambiguities resolution.

Availability problems may arise for two kind of reasons : particularly congested environments (mountains, forests, etc.) that limit the umber of satellites in view, or local shadow zones due to obstacles like bridges on a road site. In both cases, the disturbances can be minimised by choosing the optimal period of the day offering the optimal satellites constellation but this is rarely possible for heavy-duty equipment that have to work all day long. More effective improvement can be brought by systems using both GPS and GLONASS constellations or artificial satellites, so-called "pseudolites" that are already experimented here and there.

Problems and constraints linked to the base station may be reduced when real-time active reference stations networks are available. This kind of networks are already in place in several developed countries for navigation purposes, using mainly FM radio infrastructures and $\mathrm{RDS}$ coding. The extension of this principle is envisaged in some countries (Sweden, Germany, France...), with a denser network and more high-performance transmission system, for centimetre accuracy real-time applications.

\section{SOME CIVIL ENGINEERING CIC APPLICATIONS ALL OVER THE WORLD}

\subsection{Open-pit mining and earthworks}

This domain has been the first one to see effective applications of industrial CIC systems. CATERPILLAR, together with the GPS manufacturer TRIMBLE, has developed a line of such products, dedicated to big machines such as front shovels, drilling machines, wheel loaders bulldozers, scrapers, motor graders and compactors. These line is called Computer Aided Earthmoving Systems (CAES) and is based upon RTK GPS technology for positioning. Comparable products are also proposed by other companies such as Condor Earth, Aquila Mining and Modular Mining.

For instance, for ore extraction, ore grades are coloured on the on-board display and the elevation of the bucket is graphically displayed. The system has been proved to reduce the discrepancy between the operator's identification of material and actual position of the bucket from $15 \%$ to $5 \%$, thus increasing significantly the productivity and allowing a payback period less than 2 years.

\subsection{Road construction}

Road construction tasks such as asphalt spreading and compacting are also concerned by the CIC paradigm. The profitability however is more tricky to be demonstrated, given the smaller turnovers of the pieces of equipment.

Several operational sites of asphalt pavement base spreading have already been successfully executed on several sites in Europe. The positioning technology used was first the rotation laser plane combined with a odometer wheel for the plane location, for machines such as asphalt pavers. Now that Robotised Total Stations (RTS) has appeared, they tend to replace the laser plane systems, suffering from bad spatial coherency. Some novel positioning systems aims at replacing the RTS, not really designed for that application, and propose a more automated and complete positioning based upon 3D resection using rotating laser sectors and simple photo-cells as detectors (Laserguide, from the University of East London).

For asphalt compaction, the problem appears different and more simple since the tool to guide is the drums of the machine itself, to be controlled in 2D only. Here, RTK GPS-based solutions are the most appropriate ones, combined with deadreckoning sensors to get rid of local shadow zones. Several projects are being led, prefiguring commercial products for the next future, either by machinery manufacturers such as BOMAG in Germany, measuring equipment manufacturers such as GEODYNAMICS in Sweden, or European R\&D projects consortia.

The CIRC (Computer Integrated Road Construction) project $^{1}$ aims at developing a new European standard for road construction tasks. Two different but totally compatible products are under development : CIRCOM, dedicated to compaction tasks and CIRPAV For paving tasks. CIRCOM was already demonstrated successfully to the EC and to major contractors and machine manufacturers in June 1998 and January 1999. CIRPAV is currently under development and will be experimented and demonstrated at the end of 1999.

The ground sub-system of CIRC products are based upon the same kind of 3D geometrical database, and the positioning technologies chosen for the prototypes are RTK GPS + dead-reckoning for the compactor and Laserguide for the paver. Figure 6 shows a mock-up of one man-machine interface of CIRPAV, where adapted displays bring indications to the driver for controlling both altitude of the creed and trajectory of the vehicle.

\footnotetext{
${ }^{1} \mathrm{CIRC}$ is funded by the European Community under the Industrial \& Materials Technologies Programme Brite-EuRam III, $n^{\circ}$ BE-96-3039
} 


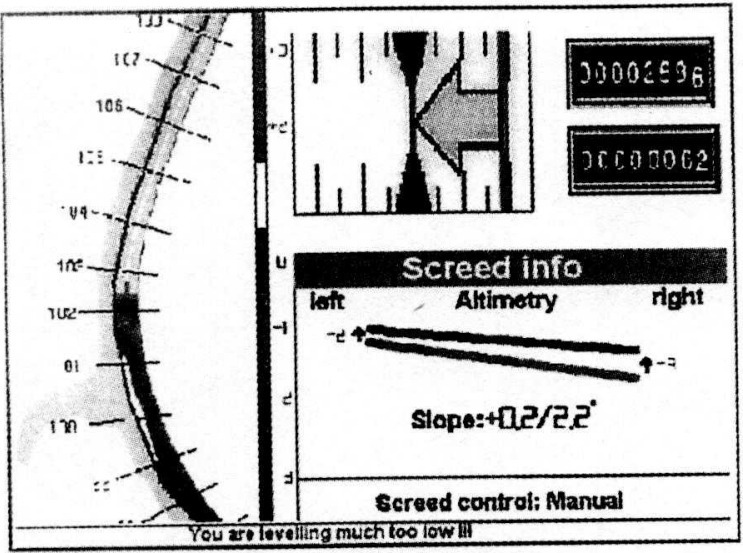

Fig. 6 : One man-machine interface of CIRPAV

\section{REFERENCES}

[1] F. Peyret, H. Philippe. "Towards Computer Integrated Road Construction" Proc. $9^{\text {th }}$ International Symposium on Automation and Robotics in Construction, pp. 859-868, Tokyo, 1992

[2] Y.J. Beliveau. "What can real-time positioning do for construction ?" Int. Journal Automation in Construction 5 (2), pp. 79-89, Elsevier, Amsterdam, 1996

[3] P. Bonnifait, F. Peyret, G. Garcia "New data fusion techniques for high-precision localization of civil-engineering equipment" Proc. $9^{\text {th }}$ International Symposium on Automation and Robotics in Construction, Pittsburgh, 1997

[4] B.J. Gorham "The Laserguide system of automatic machine guidance" Proc. $11^{\text {th }}$ International Symposium on Automation and Robotics in Construction, pp. 327-332, Brighton, 1994

[5] L.H. Pampagnin, F. Peyret, G. Garcia.

"Architecture of a GPS-based guiding system for road compaction" Proc. IEEE International Conference on Robotics and Automation (ICRA 98), Leuven, 1998

[6] F. Peyret, D. Bétaille, G. Hintzy "High-precision application of GPS in the field of real-time equipment positioning" Proc. $14^{\text {th }}$ International Symposium on Automation and Robotics for Construction, pp. 2-10, Pittsburgh, 1997 